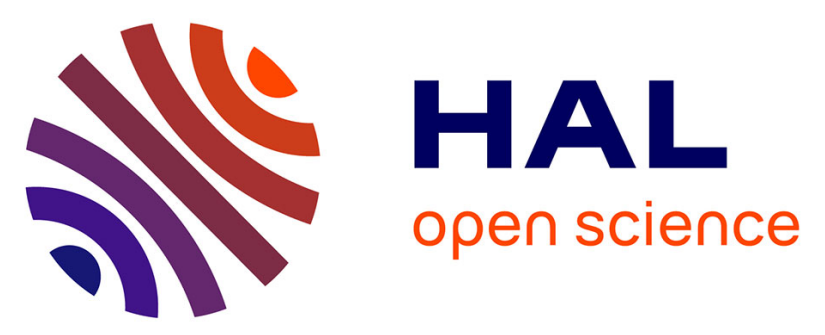

\title{
Multivariable control with grid objectives of an HVDC link embedded in a large-scale AC grid
}

Leyla Arioua, Bogdan Marinescu

\section{To cite this version:}

Leyla Arioua, Bogdan Marinescu. Multivariable control with grid objectives of an HVDC link embedded in a large-scale AC grid. International Journal of Electrical Power \& Energy Systems, 2015, 72, pp.99 - 108. 10.1016/j.ijepes.2015.02.016 . hal-01421762

\section{HAL Id: hal-01421762 \\ https://hal.science/hal-01421762}

Submitted on 30 Jan 2017

HAL is a multi-disciplinary open access archive for the deposit and dissemination of scientific research documents, whether they are published or not. The documents may come from teaching and research institutions in France or abroad, or from public or private research centers.
L'archive ouverte pluridisciplinaire HAL, est destinée au dépôt et à la diffusion de documents scientifiques de niveau recherche, publiés ou non, émanant des établissements d'enseignement et de recherche français ou étrangers, des laboratoires publics ou privés. 


\title{
Multivariable Control With Grid Objectives of an HVDC Link Embedded in a Large-Scale AC Grid
}

\author{
Leyla Arioua*1,3 and Bogdan Marinescu ${ }^{\dagger 2}$ \\ ${ }^{1}$ R\&D Division of Réseau de Transport d'Eléctricité de France, 78005 Versailles, France \\ ${ }^{2}$ IRCCyN-Ecole Centrale de Nantes, 1 rue de la Noë, 44321 Nantes cedex, France \\ ${ }^{3}$ SATIE-CNRS Laboratory of Ecole Normale Supérieure de Cachan, 94235 Cachan cedex
}

\begin{abstract}
The HVDC links are increasingly used not only to interconnect asynchronous AC systems but are also embedded into a same meshed AC power system. Thanks to its speed and flexibility, the HVDC technology is able to provide transmission system advantages as transfer capacity enhancement and power flow control. In addition, studies have shown that the way of controlling the HVDC converters impacts the stability of the AC system. This can be particularly exploited to enhance the dynamic power system performances during transients. In this paper a robust multivariable control design for HVDC link converters is proposed. It is based on the coordination of the control actions of the HVDC converters and the use of a control model which takes into account the dynamics that mostly impact stability of the neighbour zone of the HVDC link. This new methodology was used to synthesize the controller for an actual grid 1000 MW HVDC link reinforcement project called "Midi-Provence" in the southern part of the French grid. The synthesis, implementation and validation processes are presented in detail. The new controller is tested in comparison with the standard vector control. A large-scale dynamic model of the whole European power system, currently used and updated by the European TSO's for the interconnection studies has been used with Eurostag simulation software.
\end{abstract}

\section{Introduction}

The HVDC link is a mean of transmission of electric power based on high power electronics. Thanks to its speed and flexibility, the HVDC technology is able to provide the transmission system advantages as transfer capacity enhancement and power flow control [1]. Initially, it was used in power systems to interconnect asynchronous AC systems. The ends of the HVDC link are electrically independent one from each other and this avoids the propagation of perturbations between the two AC grids as it is the case of the England-France interconnection [2]. Nowadays, HVDC links are increasingly embedded into a same meshed AC system in order to enhance the grid's transmission capability and flexibility of the power system. In this context, the HVDC link co-exists with other AC system elements as for instance AC lines and generators. Several projects of insertion of this type of device in a meshed AC system are underway in Europe, as for instance France-Spain HVDC project [3], France-Italy interconnection and Midi-Provence HVDC project [4]. This latter is our case of study. In fact, a 1000 MW HVDC link will be inserted in France between the two areas Fos and Gaudière,

\footnotetext{
*leyla.arioua@yahoo.fr

†bogdan.marinescu@irccyn.ec-nantes.fr
} 
in order to enhance capacity of power transmission and the stability of the Midi-Provence region. The need of such HVDC link is due to the fact that only one single $400 \mathrm{kV}$ AC line interconnects the two areas (see Fig. 1).

It was shown that the strategy used to control the HVDC converters can impact the stability of the system in which the link is embedded [5-12]. This conducted us develop a methodology which takes into account, in a control model, the neighbour zone of the HVDC link and the dynamics that most impact transient stability. This control model captures the dynamics that are relevant for the transient stability of the power system. The controller synthesis is done on the base of this control model. As a consequence, power system performances are enhanced in addition to local performances. Preliminary results have been presente in [13]. This approach is fully developed here in order to take into account some key critical fault situations at the synthesis stage using the robust $H_{\infty}$ theory. Also the methodology is adapted to handle realistic large-scalecases as the European power system. As a mater of fact, in other papers [14,15], the transient stability is considered as an a priori objective for the synthesis of coordinated controllers for HVDC. However, the effectiveness of these methods had not been proved in the case of large-scale power systems. The proposed control is tested in comparison with the standard vector control via simulations performed with Eurostag [16]. The rest of the paper is organized as follows: in Section 2, the large-scale case used for this work is described. Then in Section 3, the method for developing the control model for the power system is presented. This control model is used in Section 4 to synthesize a robust multivariable output-feedback controller for the HVDC converters. Section 5 deals with the computation and implementation of the controller. Finally, in Section 6, simulation results are presented to illustrate the performances and the robustness of the new controller. An earlier version of this paper was published in [17].

\section{The Midi-Provence HVDC Project}

Midi-Provence project aims to strengthen the link between the two dynamic regions, Provence-AlpesCôte d'Azur and Languedoc-Roussillon by inserting an HVDC link of 1000 MW capacity between them. This two regions are now directly connected by a single $400 \mathrm{kV}$ AC line going from Tavel (Est of Gard) to La Gaudière (in the Aude) (see Fig. 1). In case of breakdown of this link, the electric power security of much of the south of France would be weakened. In some worst scenarios, this situation could lead to a major power cut in the south of France, and even in-beyond Europe, to balance supply and demand. This HVDC project responds thus to a need of enhancement of both stability and transport capacity in this region [4]. The Midi-Provence zone is considered as part of the whole European system. For the latter, we used the reference model which is maintained and generally used for interconnection studies by the European TSO's. It is a detailed non-linear model including generators along with their regulations (AVRs, PSS and Governors) and where the high voltage network $(225,400 \mathrm{kV})$ is modelled. It consists of 1121 generators, 7625 nodes, 10404 lines, 2550 transformers and 458 GVA global apparent power. The Midi-Provence HVDC link is of $230 \mathrm{~km}$ length and $1000 \mathrm{MW}$ power capacity. It is placed as shown in Fig. 1.

Note that we have considered a 2020 forecast winter situation.

\section{Control model synthesis}

The large-scale model mentioned in Section 2 cannot be used for the synthesis of the control. As a consequence, a reduced order model called a control model which must preserve the dynamics that most impact the stability of the neighbour region of the HVDC link should be extracted from the 


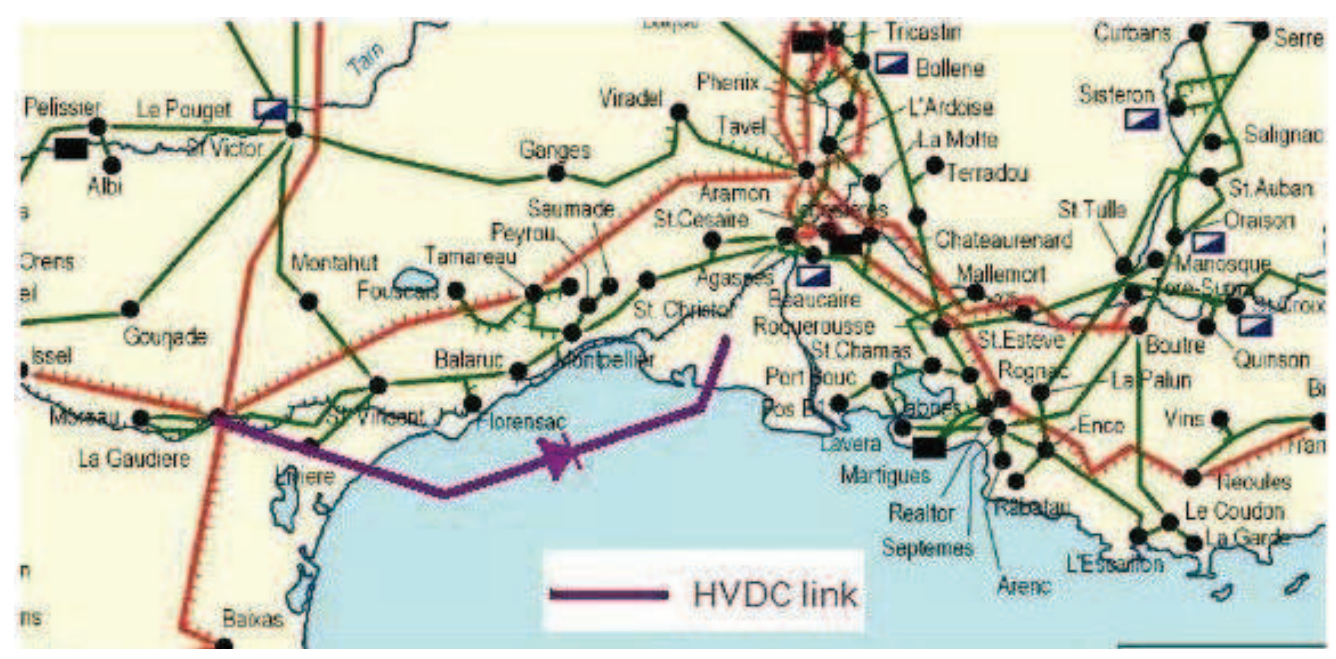

Figure 1: Midi-Provence interconnected power system

whole European model. The way to do this it is not a straightforward reduction method, but a specific methodology developed in accordance with the objectives of the control. It is detailed in the rest of the paper.

\subsection{Structure of the control model}

The control model is composed of two parts: the AC power system (grid and machines) and the HVDC link. We retain in the control model the most critical machines, i.e., those which cause the system loss of synchronism after a severe perturbation. All the other machines are skipped. Also, after this operation, the lines which no longer connect machines are considered as irrelevant topology and are also simplified.

\subsection{Construction of the control model}

\subsubsection{The study area selection}

The study area is the zone which is impacted by the HVDC link and corresponds to the neighbour zone of the HVDC interconnection of which transient stability depends on the HVDC dynamics (see Fig. 1). The extent of this zone is detected by standard stability studies usually run by TSOs.

\subsubsection{Critical machines selection strategy}

The machines retained in the control model are based on both nonlinear and linear analysis as explained below.

1. Selection on the base of CCT First, a transient stability margin of the power system is estimated by the Critical Clearing Time (CCT) which is defined as the maximal fault duration for which the system remains transiently stable [18]. The instability is then manifested by the loss of synchronism of a group of machines. The most critical machines are the ones which have the smallest CCT for a given class of faults. The latter faults are the ones put into evidence by the stability studies for the region usually run by TSOs and mentionned above. Doing so for the case Midi-Provence considered in this paper, 14 machines have been retained for the control model 
2. Selection on the base of coupling of distant machines Next, some machines external to the stability study zone may be incorporated to the control model. Indeed, electrical interaction may exist between geographically distant machines. It is well known that these interactions are characterized by inter-area modes which are of electromechanical type, i.e., they are associated to the rotors of the machines (see, e.g., [19]). Another mode which may involve geographically distant machines has been put into evidence. It is of different nature since involves only the D-axes of several distant machines and has been thus called electrical coupling modes. These both types of modes can be put into evidence by systematic modal analysis of the AC grid. The list defined at step 1 for the machines to be retained for the control model in the initial neighbour zone of the HVDC link for which the transient stability analysis (CCT evaluation) has been performed, has to be enriched by the machines outside this zone but which are involved in the coupling modes mentioned above together with the machines found at step 1.

\subsubsection{Reduction of the topology}

Reduction of the topology of the power system is also performed. The buses to which the retained machines are connected are kept along with the neighbour zone buses. The rest of the buses and branches are replaced by a WARD-PV method [20], i.e., by equivalent impedances and injectors.

\subsubsection{Perturbed models}

The model described above can be considered as nominal in the sense that it corresponds to the no incident situation of the power system. A family of perturbed models was also generated. Each member of this family corresponds to a model under a critical fault situation. The electric system is then considered as a set of perturbed systems. The most severe fault generates the the worst-case situation (see Section 4.3).

\subsection{Modelling of the VSC-HVDC}

A voltage-source converters (VSC) based HVDC is considered as in Fig. 2a. The converters are VSC employing IGBT power semiconductors, one operating as a rectifier and the other as an inverter. The two converters are connected through a DC cable. These converters have the ability to rapidly control the transmitted active power, and also to independently exchange reactive power with the AC system at each end. The high frequency switching operation of the power electronics is neglected and each converter can be considered as an ideal sinusoidal voltage source whose magnitude $U_{c}$ and phase angle $\theta$ can be controlled (see [21]). The VSC-HVDC is thus modelled as two sources, each one in series with the converter transformer (see Fig. $2 \mathrm{~b}$ where $\bar{U}_{c 1}=U_{c 1 i}+j U_{c 1 j}, \bar{U}_{c 2}=U_{c 2 i}+j U_{c 2 j}$ and $\theta_{1}, \theta_{2}$ are, respectively, the magnitudes and the phase angles of the voltage sources, $P_{1}, P_{2}$, and, $Q_{1}, Q_{2}$ are, respectively, active and reactive powers exchanged with the power system and $X_{T 1}, X_{T 2}$ are the transformer reactances). In this model of HVDC, it is assumed that the DC voltage is kept close to its rated voltage, because its dynamic is much faster than the one of currents. Therefore, the losses of the converters are assumed constant, regardless of the current through the converters. They are represented as a constant active load. However, the losses of the DC cables are neglected [21]. This leads to

$$
\begin{aligned}
& P_{1}=\left(U_{1}\left(\sin \theta_{1} U_{c 1 i}-\cos \theta_{1} U_{c 1 j}\right)\right) / X_{T 1} \\
& Q_{1}=\left(U_{1}^{2}-U_{1}\left(\cos \theta_{1} U_{c 1 i}-\sin \theta_{1} U_{c 1 j}\right)\right) / X_{T 1} \\
& P_{2}=\left(U_{2}\left(\sin \theta_{2} U_{c 2 i}-\cos \theta_{2} U_{c 2 j}\right)\right) / X_{T 2} \\
& Q_{2}=\left(U_{2}^{2}-U_{2}\left(\cos \theta_{2} U_{c 2 i}-\sin \theta_{2} U_{c 2 j}\right)\right) / X_{T 2} .
\end{aligned}
$$




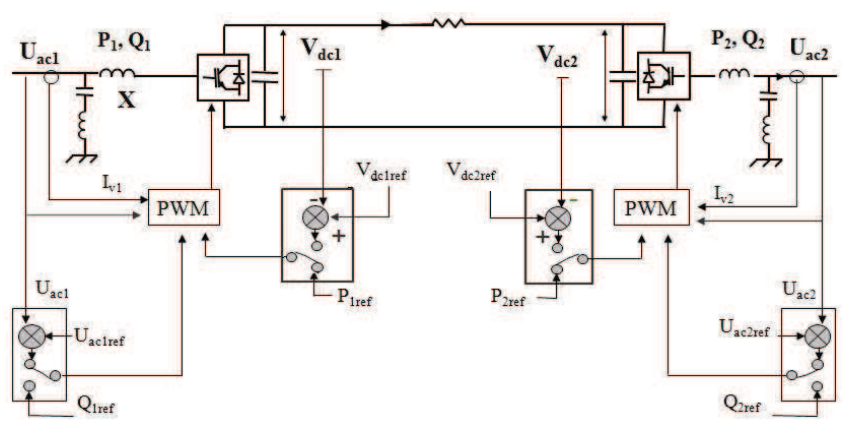

(a) Scheme of a detailed VSC-HVDC

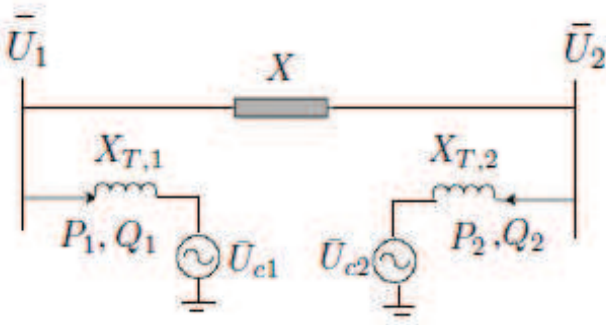

(b) Simplified model of VSC-HVDC

Figure 2: VSC-HVDC link

In addition, notice that $P_{2}=-P_{1}$. The control variables can be expressed as follows:

$$
\begin{aligned}
& U_{c 1 i}=U_{c 1 i 0}+\Delta U_{c 1 i}, U_{c 1 j}=U_{c 1 j 0}+\Delta U_{c 1 j}, \\
& U_{c 2 i}=U_{c 2 i 0}+\Delta U_{c 2 i}, U_{c 2 j}=U_{c 2 j 0}+\Delta U_{c 2 j}
\end{aligned}
$$

\subsection{Validation of the control model}

The resulting control model is validated by non-linear simulations comparing responses of the reduced and the full model when a fault occurred near the HVDC link. Theses simulations were done using Eurostag software. Fig. 3a and Fig. 3b for instance, illustrate the speed responses of two generators retained in the control model $G_{1}$ and $G_{2}$. We can observe that the two responses of the control model and the full model are closed to each other for this two generators.

\section{Synthesis of the control law}

In our previous work [13], an output-feedback controller for HVDC converters has been developed using an LQ pole placement [13]. In this present work an H-infinity control theory is used to take into account not only the nominal model of the system, but also the family of disturbed models presented in Section 3.2.4. This allowed us to improve both performances and robustness which allows a more robust control design.

\subsection{Design Requirements}

The synthesis of the HVDC controller is done such that the performances are ensured for several cases of fault (i.e, the enhancement of transient stability) along with local ones which are tracking of references for active power, reactive power. For these purpose, a robust multivariable control is applied.

To avoid the use of remote variables (e.g., machines speed, angles, ...), an output feedback structure described in Fig. 4 is used. Only variables available at converter stations are used and a coordinated controller is developed for both converters. A linear approximation of the control model around an equilibrium point is used for the synthesis.

\subsection{Linear approximation of the control model}

The control model includes the machines retained in the control model and their regulations (AVRs and governors) be represented by the non linear differential and algebraic equations 


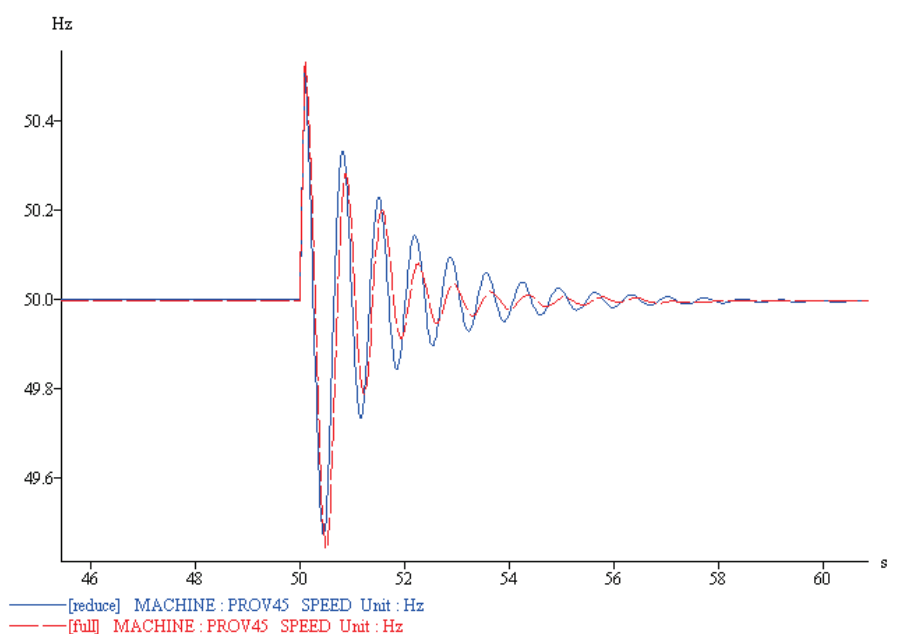

(a) Speed response of G1 in the two cases of full and reduced models (p.u)

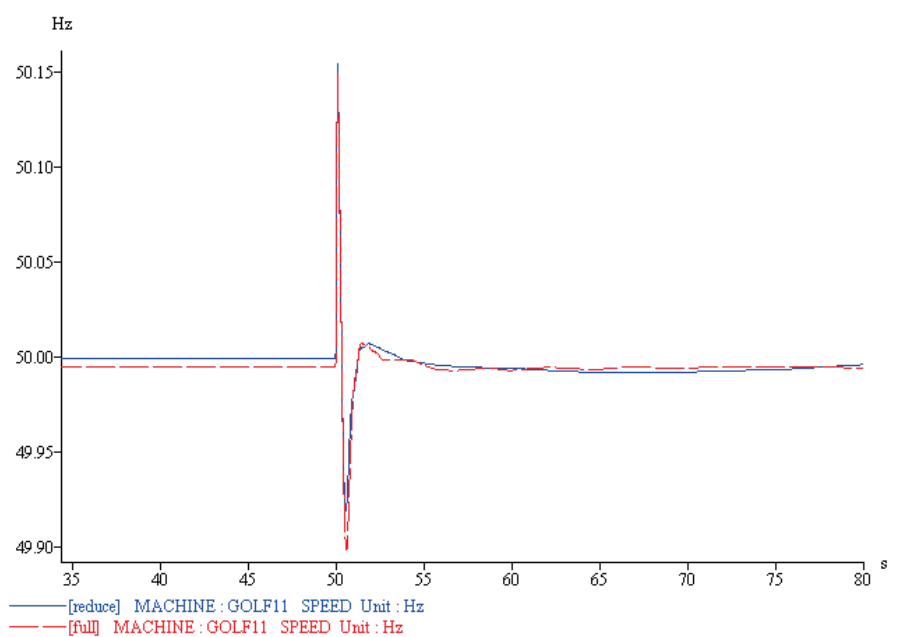

(b) Speed response of the G2 in the two cases of full and reduced models

Figure 3: Speed responses to a $100 \mathrm{~ms}$ short-circuit in the two cases of full and reduced models (p.u)

$$
\begin{aligned}
& \dot{x}=f(x, U) \\
& y=g(x, U)
\end{aligned}
$$

where $\boldsymbol{x}$ represents the state variables (speeds, angles of machines, AVRs and governors variables ...) of the machines retained in the control model, $U$ the control variables, $\boldsymbol{y}$ the outputs and let $y_{\text {ref }}$ be the control references :

$U=\left[U_{c 1 i}, U_{c 1 j}, U_{c 2 i}, U_{c 2 j}\right]$

$y=\left[P_{1}, Q_{1}, Q_{2}\right]$.

$y_{\text {ref }}=\left[P_{r e f 1}, Q_{r e f 1}, Q_{r e f 2}\right]$. 


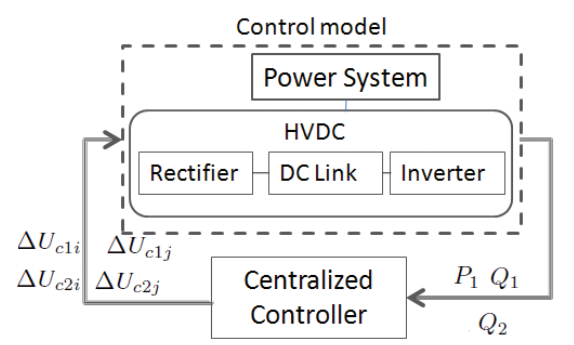

Figure 4: Structure of the output-feedback control

The linearization of the system (3) around a given equilibrium point $\left(x_{0}, U_{0}\right)$ is

$$
\Sigma:\left\{\begin{aligned}
\Delta \dot{x} & =A \Delta x+B \Delta U \\
\Delta y & =C \Delta x
\end{aligned}\right.
$$

where $\Delta x=x-x_{0}, \Delta U=\left[\Delta U_{c 1 i}, \Delta U_{c 1 j}, \Delta U_{c 2 i}, \Delta U_{c 2 j}\right], \Delta y=y-y_{0}$ and $A, B, C$ are constant matrices.

The model of the European power system is of a very large scale (17020 sate variables and 43940 total variables). Thanks to the control model developed in Section 3, we can reduce the order of the model considered for the control to 310 state variables with a total of 1117 variables. By performing a minimal realization using Robust control toolbox of Matlab, a 30th-order control model is obtained. Notice that dedicated tools (like, e.g, Eurostag [16]), provide the linear model (4) for large-scale power systems.

\subsection{Mixed sensitivity $\mathrm{H}$-infinity control design}

Using the nominal model $P(s)$ developed in Section 3 and the set of the linearised perturbed models described in Section 3.2.4, an uncertain model $P_{\Delta}(s)$ is generated and is expressed as $P_{\Delta}(s)=$ $\left(1+\Delta(s) W_{t}(s)\right) P(s)$ as shown in Fig. 5 where :

- $\Delta(s)$ : is the normalized model deviation with respect to the nominal model.

- $W_{t}(s)$ : is the uncertainty weight and is used to capture how the relative uncertainty varies with frequency.

The Fig. 6 shows the singular values (frequency responses for multivariable systems) of the deviation of all the perturbed models with respect to the nominal one and the weight $W_{t}(s)$.

The uncertain model generated above leads us to develop a controller taking into consideration the worst-case situation. This can be achieved using some methods existing in the control theory (for example $\mu$ synthesis, $H_{\infty}$ control). In this paper the $H_{\infty}$ control and more precisely the Mixed sensitivity $H$-infinity control design is used. The $H_{\infty}$ problem formulation and control law computation is recalled in the Appendix.

Let the sensitivity $S(s)=(I+P(s) K(s))^{-1}$ be the closed loop transfer function matrix from the reference inputs to the error and the complementary sensitivity $T(s)=P(s) K(s)(I+P(s) K(s))^{-1}$ be the closed-loop transfer function matrix from the references to the measured outputs where $\mathrm{P}$ is the nominal model, $\mathrm{K}$ the controller, and I identity matrix.

The principle of this control design is that if it exists a controller $K(s)$ given by its transfer matrix 


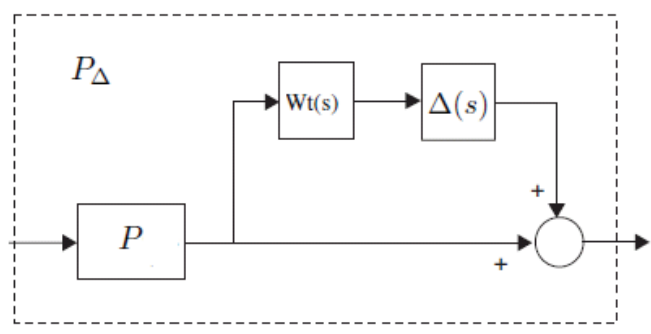

Figure 5: Plant with multiplicative output uncertainty

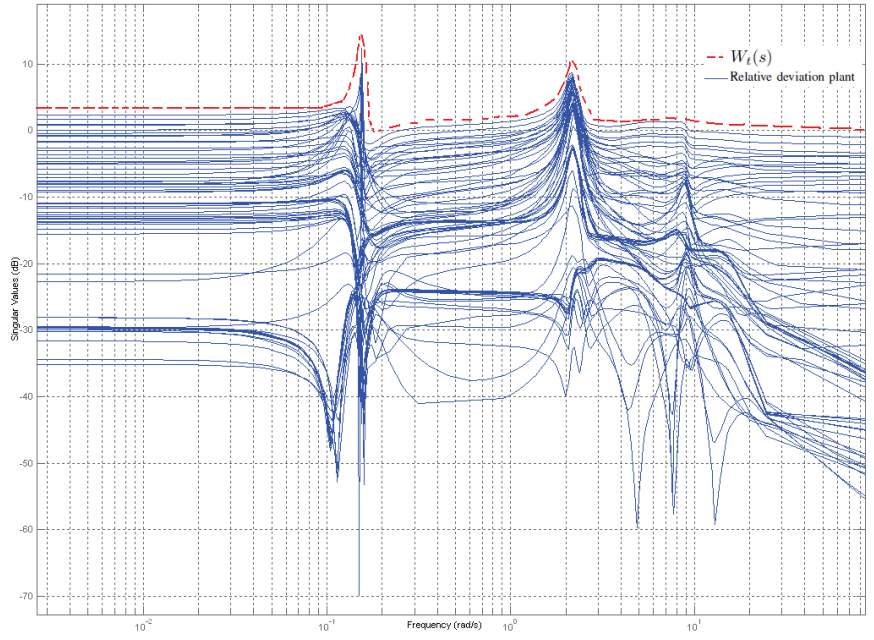

Figure 6: Relative gaps vs. Magnitude of Wt(s) 


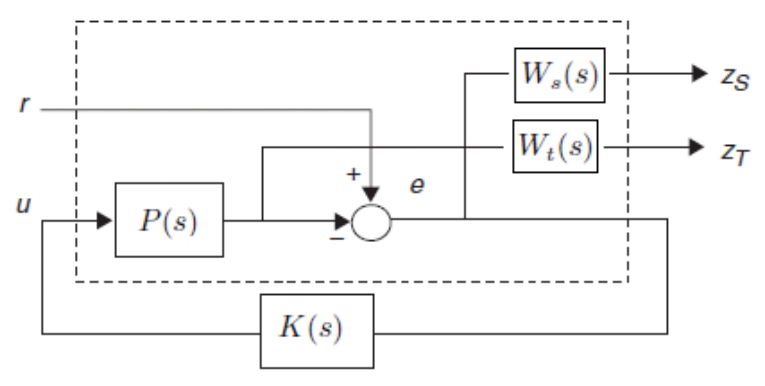

Figure 7: Block diagram of the generalized plant in the mixed H-infinity synthesis [22]

which ensures $\left\|W_{t}(s) T(s)\right\|_{\infty} \leq 1$ for all frequencies, the closed-loop system is ensured stable for all plants in $P_{\Delta}$ (see [22]).

The desired performances are achieved if $\left\|W_{s}(s) S(s)\right\|_{\infty} \leq 1 . W_{s}(s)$ and $W_{t}(s)$ are the weights used to shape the sensitivity and the complementary sensitivity respectively. The weight $W_{t}(s)$ is used to ensure robustness against model uncertainties. The weight $W_{s}(s)$ is based on shaping the sensitivity for desired tracking performances.

The state space model is augmented with the matrix weights $W_{t}(s)$ and $W_{s}(s)$ and form the augmented system $G(s)$ (described in the Appendix) as shown in Fig. 7.

The objective of the mixed sensitivity problem is to find a stabilizing controller $K$ which minimizes.

$$
\left\|\left[\begin{array}{l}
W_{s}(s) S(s) \\
W_{t}(s) T(s)
\end{array}\right]\right\|_{\infty}
$$

In this work $W_{t}(s)$ is the uncertainty weight and $W_{s}(s)$ is chosen in the form (see [23])

$$
W_{s}(s)=\left(\frac{s / \sqrt[k]{M_{s}}+\omega_{b}}{s+\omega_{b} \sqrt[k]{\varepsilon}}\right)^{k}
$$

where $\omega_{b}$ is the bandwidth, $M_{s}$ the peak sensitivity, $\varepsilon$ the steady state error desired with respect to a step input, and $k$ is the order of the weighting function. Notice that making $\varepsilon$ small enforce the integral action (in our case $\varepsilon=10^{-4}$ )

\section{Computation and implementation of the controller}

The robust multivariable controller $K(s)$ responding to the mixed sensitivity problem formulated in Section 4 is calculated by solving the Ricatti equation as it is described in the Appendix. In practice, a function of Robust control toolbox of Matlab is used to solve the problem. The final controller is given in its state representation $\left(A_{K}, B_{K}, C_{K}, D_{K}\right)$ and it is of the same order as the plant model augmented by the weights $W_{s}$ and $W_{t}$. The model obtained after reduction and minimal realization is of 30th-order. The controller obtained is of 40th-order which is too high for the implementation. To deal with this problem, a reduction of the controller order is envisaged. A reduction method based on the singular values [23] helped us to generate a controller of 5th-order. The truncations has been performed with some existing functions in Robust control design toolbox of Matlab.

This resulating controller can be written in its matrix transfer function form:

$K(s)=C_{K}\left(s I-A_{K}\right)^{-1} B_{K}$, 
where $A_{K}$ is a state matrix of 5 th order.

\section{A coordinated structure}

The controller transfer matrix obtained above can be detailed

$$
\begin{gathered}
\left(\begin{array}{c}
\Delta \boldsymbol{U}_{1} \\
\Delta \boldsymbol{U}_{2}
\end{array}\right)=\underbrace{\left(\begin{array}{ll}
\boldsymbol{K}_{11}(s) & \boldsymbol{K}_{12}(s) \\
\boldsymbol{K}_{21}(s) & \boldsymbol{K}_{22}(s)
\end{array}\right)}_{\boldsymbol{K}(s)}\left(\begin{array}{c}
\Delta \boldsymbol{y}_{1} \\
\Delta \boldsymbol{y}_{2}
\end{array}\right) \\
\Delta \boldsymbol{U}_{1}=\left(\begin{array}{c}
\Delta U_{c 1 i} \\
\Delta U_{c 1 j}
\end{array}\right), \Delta \boldsymbol{U}_{2}=\left(\begin{array}{c}
\Delta U_{c 2 i} \\
\Delta U_{c 2 j}
\end{array}\right)
\end{gathered}
$$

and

$$
\Delta \boldsymbol{y}_{1}=\left(\begin{array}{c}
\Delta P_{1} \\
\Delta Q_{1}
\end{array}\right), \Delta \boldsymbol{y}_{2}=\left(\Delta Q_{2}\right) .
$$

The transfer matrices $K_{12}(s)$ and $K_{21}(s)$ are the cross-terms of the controller and ensure the coordination between the control of the two HVDC converters. In the standard vector control, HVDC links are controlled with a decentralized structure, which is equivalent to consider a priori that the two HVDC converters don't interact $\left(K_{12}(s)=0\right.$ and $\left.K_{21}(s)=0\right)$. This hypothesis is not valid when the HVDC is used in the new grid context mentioned in the introduction.

Each term of the $K_{i j}$ transfer matrix is in the form of :

$$
k_{i j}=\frac{\alpha_{i j}}{s} \prod_{n=1}^{4} \frac{1+\tau_{n} s}{1+\gamma_{n} s}
$$

In Our case of study the controller is given in the Table 1. One can observe that it is of 5th-order which is due to the reduction of the controller as we have explained above.

\section{Simulation tests}

This section illustrates the effectiveness of the H-infinity controller proposed. Simulation tests using Eurostag software [16] were performed. The new synthesized controller is tested on the full model introduced in Section 2. An export of $1000 \mathrm{MW}$ is considered from Fos to Gaudière via the DC link. The robust H-infinity controller is compared with the standard vector controller (see for example [24]) which has a cascade structure with an inner loop more rapid than the outer one. The proportional and integral gains of this control are synthesized using standard criteria for electrical drives. The two controllers are tuned to satisfy almost the same performance specifications (the usual time setting for HVDC voltage and power control).

\subsection{Transient stability}

In a first scenario, a symmetrical double fault at Tavel-Tamareau, cleared after $100 \mathrm{~ms}$ by opening the lines at their both ends is simulated. Fig. 9a and Fig. 9b show the responses of active and reactive powers exported. It can be observed that the dynamic responses for this case of fault are better with the new controller especially for the reactive power. Indeed, more reactive power is needed with the vector control. Moreover, a limitation is reached in this case during the transient.

In a second scenario, a symmetrical short-circuit is applied at one of the HVDC terminals (in the Fos 
Table 1: Transfer functions of the controller $K(s)$

\begin{tabular}{|l|c|}
\hline & Transfer \\
function \\
\hline$k_{11}$ & $\frac{80.83}{s} \frac{(s+0.018)}{(s+0.06)} \frac{(s+0.012)}{(s+0.03)} \frac{\left(s^{2}+0.03 s+5.2410^{-3}\right)}{\left(s^{2}+0.011 s+3.1210^{-3}\right)}$ \\
\hline$k_{21}$ & $\frac{-18.42}{s} \frac{(s+0.019)}{(s+0.06)} \frac{(s+0.101)}{(s+0.03)} \frac{\left(s^{2}+0.05 s+6.310^{-3}\right)}{\left(s^{2}+0.011 s+3.1210^{-3}\right)}$ \\
\hline$k_{31}$ & $\frac{-1.0469}{s} \frac{(s+0.028)}{(s+0.06)} \frac{(s+0.02)}{(s+0.03)} \frac{\left(s^{2}+0.035 s+0.58010^{-2}\right)}{\left(s^{2}+0.011 s+3.1210^{-3}\right)}$ \\
\hline$k_{41}$ & $\frac{5.74}{s} \frac{(s+0.051)}{(s+0.06)} \frac{(s+0.042)}{(s+0.03)} \frac{\left(s^{2}+0.079 s+6.110^{-3}\right)}{\left(s^{2}+0.011 s+3.1210^{-3}\right)}$ \\
\hline$k_{12}$ & $\frac{11.863}{s} \frac{(s+0.3012)}{(s+0.06)} \frac{(s+0.15)}{(s+0.03)} \frac{\left(s^{2}+0.09 s+4.110^{-3}\right)}{\left(s^{2}+0.011 s+3.1210^{-3}\right)}$ \\
\hline$k_{22}$ & $\frac{-80.04}{s} \frac{(s+0.0412)}{(s+0.06)} \frac{(s+0.012)}{(s+0.03)} \frac{\left(s^{2}+0.010 s+8.3210^{-3}\right)}{\left(s^{2}+0.011 s+3.1210^{-3}\right)}$ \\
\hline$k_{32}$ & $\frac{-0.68032}{s} \frac{(s+0.01)}{(s+0.06)} \frac{(s+0.0048)}{(s+0.03)} \frac{\left(s^{2}+0.08 s+5.1210^{-3}\right)}{\left(s^{2}+0.011 s+3.1210^{-3}\right)}$ \\
\hline$k_{42}$ & $\frac{-3.565}{s} \frac{(s+0.012)}{(s+0.06)} \frac{(s+0.0054)}{(s+0.03)} \frac{\left(s^{2}+0.021 s+4.8910^{-3}\right)}{\left(s^{2}+0.011 s+3.1210^{-3}\right)}$ \\
\hline$k_{13}$ & $\frac{2.56}{s} \frac{(s+0.075)}{(s+0.06)} \frac{(s+0.012)}{(s+0.06)} \frac{\left(s^{2}+0.022 s+4.510^{-3}\right)}{\left(s^{2}+0.011 s+3.1210^{-3}\right)}$ \\
\hline$k_{23}$ & $\frac{2.6}{s} \frac{(s+0.062)}{(s+0.06)} \frac{(s+0.075)}{(s+0.03)} \frac{\left(s^{2}+0.041 s+6.210^{-3}\right)}{\left(s^{2}+0.011 s+3.1210^{-3}\right)}$ \\
\hline$k_{33}$ & $\frac{-5.94}{s} \frac{(s+0.050)}{(s+0.06)} \frac{(s+0.02)}{(s+0.03)} \frac{\left(s^{2}+0.078 s+510^{-3}\right)}{\left(s^{2}+0.011 s+3.1210^{-3}\right)}$ \\
\hline$k_{43}$ & $\frac{-84.52}{s} \frac{(s+0.031)}{(s+0.06)} \frac{(s+0.10)}{(s+0.03)} \frac{\left(s^{2}+0.009 s+4.3110^{-3}\right)}{\left(s^{2}+0.011 s+3.1210^{-3}\right)}$ \\
\hline
\end{tabular}

area). As for the previous case the transient responses are enhanced in comparison to the standard vector control. This is confirmed by analysis of the transient stability of the zone. In fact, Table 2 shows that the critical clearing time are augmented when the new control is used.

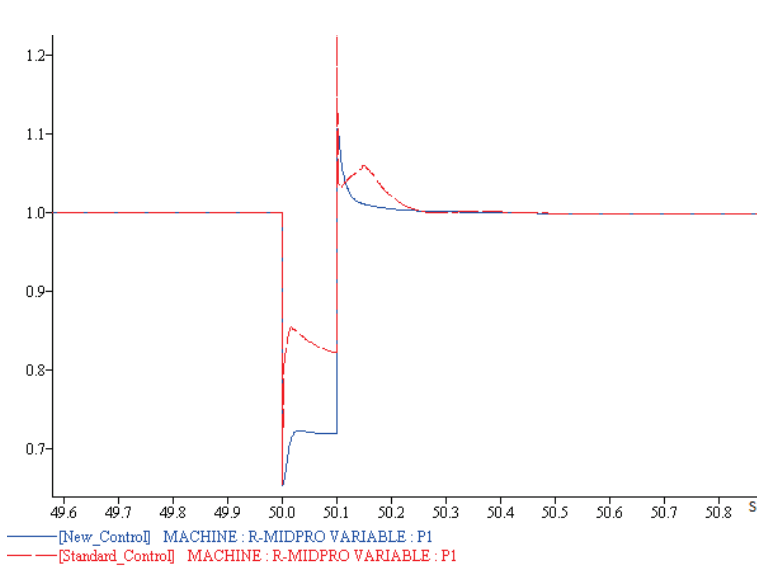

(a) Response of the active power $P_{1}$ (p.u)

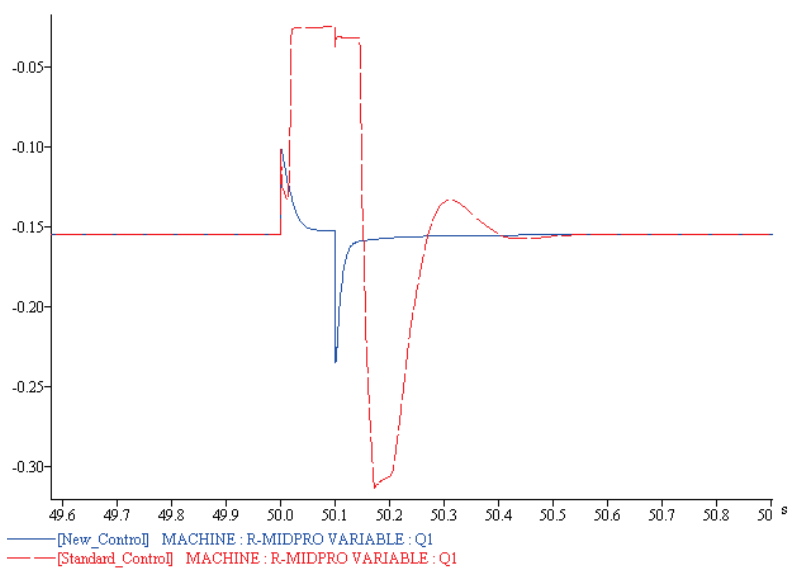

(b) Response of the reactive power $Q_{1}$ (p.u)

Figure 8: Responses to a 100 ms Tavel-Tamareau short-circuit 


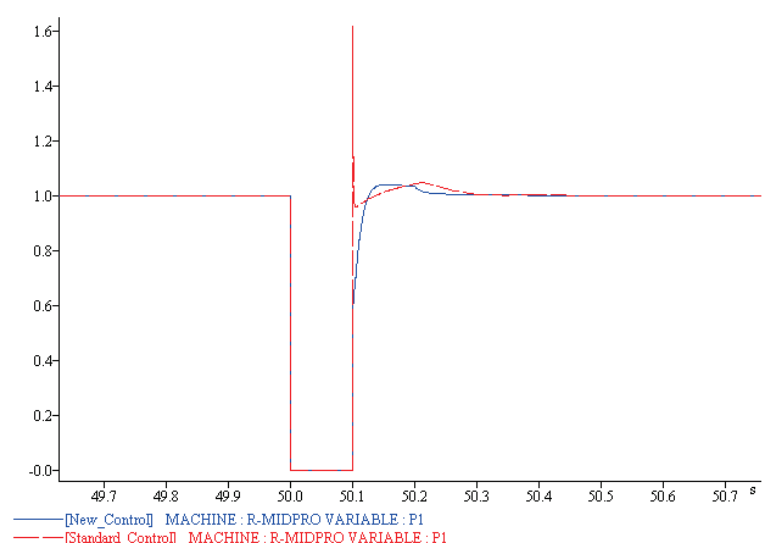

(a) Response of the active power $P_{1}$ (p.u)

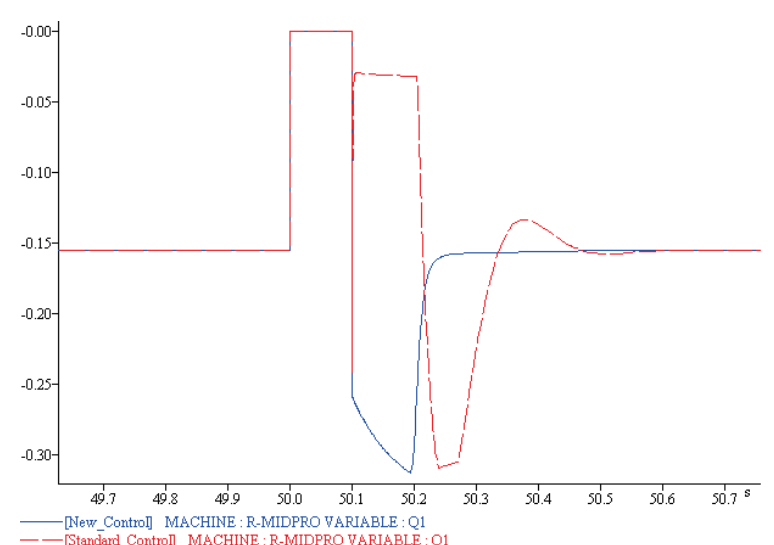

(b) Response of the reactive power $Q_{1}$ (p.u)

Figure 9: Responses to a $100 \mathrm{~ms}$ short-circuit near Fos area in case of $1000 \mathrm{MW}$ power export

Table 2: Critical clearing time validation of the two controllers

\begin{tabular}{|c|c||c||}
\hline $\begin{array}{c}\text { Fault } \\
\text { simulated }\end{array}$ & $\begin{array}{c}\text { Standard } \\
\text { controller } \\
\text { CCT }[\mathrm{ms}]\end{array}$ & $\begin{array}{c}\text { New } \\
\text { controller } \\
\text { CCT [ms] }\end{array}$ \\
\hline fault 1 & 216 & 224 \\
fault 2 & 192 & 204 \\
fault 3 & 183 & 195 \\
fault 4 & 181 & 192 \\
fault 5 & 194 & 205 \\
\hline
\end{tabular}

\subsection{Robustness against variation of the operating point}

The proposed controller was synthesized using the linearised model. This lead us to perform some robustness tests against variation of the operating point of the HVDC link. We have considered the situation of a $450 \mathrm{MW}$ import of active power from Gaudière area to Fos. As for the previous tests, a $100 \mathrm{~ms}$ symmetrical fault is applied at one of the HVDC terminals (Gaudière area). The active and reactive powers imported are represented in Fig. 10a and Fig. 10b and both are comparable with the ones obtained when the export scenario is considered (Fig. 9a and Fig. 9b). This confirm the good robustness of the performances of the proposed controller against variation of operating conditions.

\section{Conclusion}

A new method for synthesizing the controllers of HVDC links has been presented. It is based on the use of a control model which takes into account the region of the power system neighbouring the HVDC link and impacted by it. The main advantage of this new methodology is that the main dynamics of the full power system are taken into account via a control model. It has been shown that this strategy can be applied to a realistic large scale power system.

Moreover, an H-infinity control design has been used. It allowed us to consider at the synthesis stage some faulted situations and this contributed to enhance robustness along with performances of 


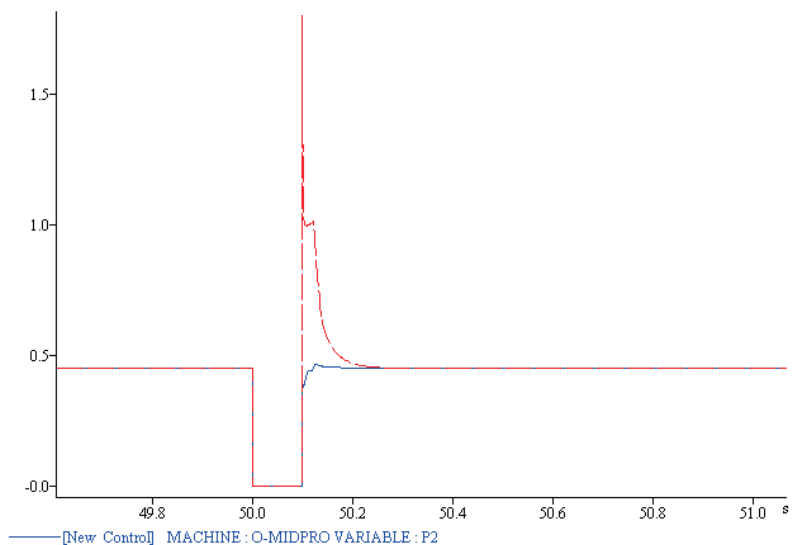

(a) Response of the active power $P_{2}$ (p.u)

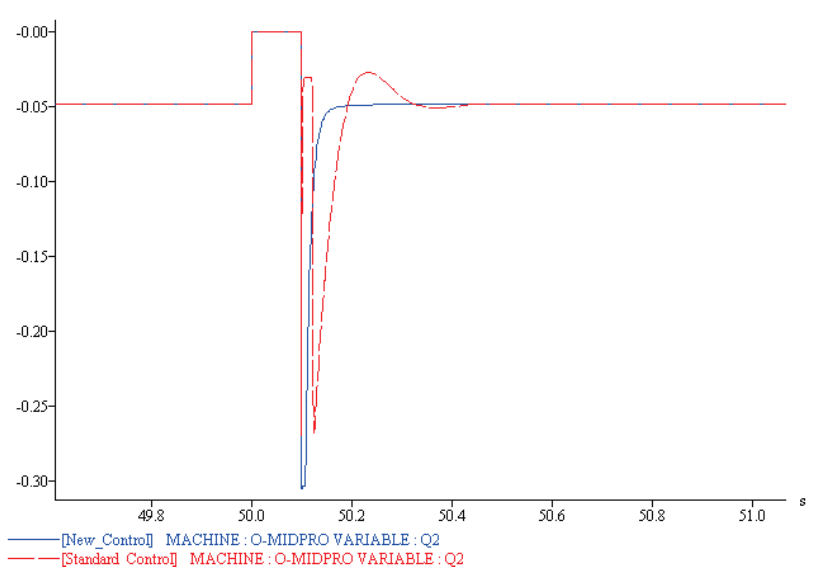

(b) Response of the reactive power $Q_{2}$ (p.u)

Figure 10: Responses to a $100 \mathrm{~ms}$ short-circuit near Gaudière area in case of $450 \mathrm{MW}$ power import

the HVDC link controller and a better fault recovery.

The control model proposed in this methodology is a way to connect the system expertise to the control one. More specially, in more cases, the manufactures do not possess the knowledge and the data to construct such a model and, as a consequence, the control of the DC link is synthesized with a very simplified model of the surrounding system. Most of the time, infinite buses or large power generators are considered connected to the two ends. The control model proposed here is rich enough to improve the performances of the controllers and simple enough to be built and provided by TSO's to the manufactures.

\section{Acknowledgment}

The authors would like to thank Prof. Eric Monmasson, University of Cergy-Pontoise and Dr. Alexandre Parisot, head of Integration of New Technology division (INT) of RTE for their helpful suggestions and remarks.

\section{Appendix: Solution of the $H_{\infty}$ control problem}

\subsection{The system's representation}

The stationary linear system can be given by its state representation :

$$
\left\{\begin{array}{l}
\dot{x}(t)=A x(t)+B u(t) \\
y(t)=C x(t)+D u(t)
\end{array}\right.
$$

The transfer matrix of this system is

$$
P(s)=C(s I-A)^{-1} B+D
$$

and is noted $P=\left[\begin{array}{c|c}A & B \\ \hline C & D\end{array}\right]$.

The controller $K(s)$ and the uncertainties of $G(s)$ can be put into evidence according to Figure 11. 
where

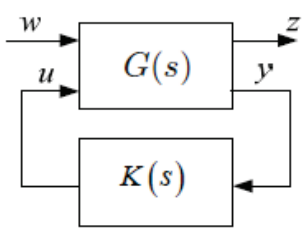

With controller

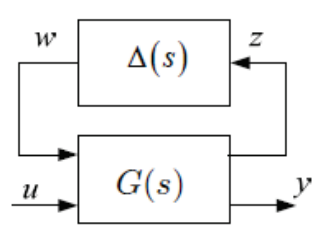

With uncertainties

Figure 11: Standard form representation of a system

$G(s)$ : the generalized process (the process $P(s)$ augmented with the weights)

$K(s)$ : the controller.

$\Delta(s)$ : the uncertainty modelling

$w$ : exogenous entries of the system

$z$ : errors between references and outputs of the system

$y$ : measured outputs

$u$ : the control signals

This representation is called Standard form of the system and generates an augmented system described by a differential equation and two output equations :

$$
\begin{gathered}
\left\{\begin{array}{l}
\dot{x}(t)=A x(t)+B_{1} w(t)+B_{2} u(t) \\
z(t)=C_{1} x(t)+D_{11} w(t)+D_{12} u(t) \\
y(t)=C_{2} x(t)+D_{21} w(t)+D_{22} u(t)
\end{array}\right. \\
G=\left[\begin{array}{c|cc}
A & B_{1} & B_{2} \\
\hline C_{1} & D_{11} & D_{12} \\
C_{2} & D_{21} & D_{22}
\end{array}\right],
\end{gathered}
$$

The transfer matrix $G(s)$ of this augmented system can be partitioned according to the inputs/outputs:

$$
G(s)=\left[\begin{array}{ll}
G_{11}(s) & G_{12}(s) \\
G_{21}(s) & G_{22}(s)
\end{array}\right] .
$$

\subsection{Problem formulation}

The plant $G$ has two inputs, the exogenous input $w$, that includes reference signal and disturbances, and the control variables $u$. There are two outputs, the error signals $z$ that we want to minimize, and the measured variables $y$, that we use to control the system. $y$ is used in $K$ to calculate the control variable $u$.

It is possible to express the dependency of $\mathrm{z}$ on $\mathrm{w}$ as: $z=F_{l}(P, K) w$,

$$
F_{l}(P, K)=G_{11}+G_{12} K\left(I-G_{22} K\right)^{-1} G_{21},
$$

called the lower linear fractional transformation. 
The objective of the $H_{\infty}$ control design is to find a controller $K$ which stabilizes the closed loop for all the plants in the family $P_{\Delta}(s)$ and such that $F_{l}(P, K)$ is minimised according to the $H_{\infty}$ norm. The infinity norm of the transfer function matrix is defined as:

$\left\|F_{l}(P, K)\right\|_{\infty}=\sup _{\omega} \bar{\sigma}\left(F_{l}(P, K)(j \omega)\right)$ where $\bar{\sigma}$ is the maximum singular value of the matrix $F_{l}(P, K)(j \omega)$. Since the $H_{\infty}$ norm to be minimized is a superior value over $\omega$ of the maximum singular value $\bar{\sigma}$ of the transfer $F_{l}(P, K)(j \omega)$, this allows us to develop a controller which takes into consideration the worst-case situation.

Notice that the singular values are used for in the case of multivariable systems to represent the model gains over all input and output directions.

\subsection{Solution of the $H_{\infty}$ control problem}

Consider the generalized state representation described by (13), (14) and its transfer matrix given by (15). We assume that $D_{22}=0$, i.e., it don't exist any direct transmission from the input to the output.

The solution of the problem is based on the solution of the algebraic Riccati equation [23]

$$
X E+E^{T} X-X W X+Q=0,
$$

with $W=W^{T}$ and $Q=Q^{T}$.

A stabilizing solution $X$ of (17), if it exists, is a symmetrical matrix such that $(E-W X)$ is a stable matrix (eigenvalues with strictly negative real parts). This solution is noted

$$
X=\operatorname{Ric}\left(\begin{array}{cc}
E & -W \\
-Q & -E^{T}
\end{array}\right)
$$

The solution of the Riccati equation :

Let be $R_{n}=D_{1 *}^{T} D_{1 *}-\left[\begin{array}{cc}\gamma^{2} I_{m 1} & 0 \\ 0 & 0\end{array}\right] \quad$ and $\quad \tilde{R}_{n}=D_{* 1} D_{* 1}^{T}-\left[\begin{array}{cc}\gamma^{2} I_{p 1} & 0 \\ 0 & 0\end{array}\right]$.

where $D_{1 *}=\left[\begin{array}{ll}D_{11} & D_{12}\end{array}\right]$ and $D_{* 1}=\left[\begin{array}{l}D_{11} \\ D_{21}\end{array}\right]$.

Since $R_{n}$ and $\tilde{R}_{n}$ are not singular, we define the two Hamiltonian matrices

$$
\begin{gathered}
H=\left[\begin{array}{cc}
A & 0 \\
-C_{1}^{T} C_{1} & -A^{T}
\end{array}\right]-\left[\begin{array}{c}
B \\
-C_{1}^{T} D_{1 *}
\end{array}\right] R_{n}^{-1}\left[\begin{array}{ll}
D_{1 *}^{T} C_{1} & B^{T}
\end{array}\right] \\
J=\left[\begin{array}{cc}
A^{T} & 0 \\
-B_{1} B_{1}^{T} & -A
\end{array}\right]-\left[\begin{array}{c}
C^{T} \\
-B_{1} D_{* 1}^{T}
\end{array}\right] \tilde{R}_{n}^{-1}\left[\begin{array}{ll}
D_{* 1} B_{1}^{T} & C
\end{array}\right] .
\end{gathered}
$$

If we put $X=\operatorname{Ric}(H)$ and $Y=\operatorname{Ric}(J)$, we can define the two matrices :

$$
\begin{gathered}
F=-R_{n}^{-1}\left(D_{1 *}^{T} C_{1}+B^{T} X\right)=\left[\begin{array}{c}
F_{1} \\
F_{2}
\end{array}\right]=\left[\begin{array}{c}
F_{11} \\
F_{12} \\
F_{2}
\end{array}\right] \\
L=-\left(B_{1} D_{* 1}^{T}+Y C^{T}\right) \tilde{R}_{n}^{-1}=\left[\begin{array}{ll}
L_{1} & L_{2}
\end{array}\right]=\left[\begin{array}{lll}
L_{11} & L_{12} & L_{2}
\end{array}\right],
\end{gathered}
$$

where $F_{1}, F_{2}, F_{11}$ and $F_{12}$ are formed respectively by $m_{1}, m_{2}, m_{1}-p_{2}$ and $p_{2}$ lines and $L_{1}, L_{2}, L_{11}$ $L_{12}$ are formed respectively by $p_{1}, p_{2}, p_{1}-m_{2}$ and $m_{2}$ columns.

A solution exists if the two following conditions are satisfied : 
1. $\left(A, B_{2}\right)$ is stabilizable and $\left(C_{2}, A\right)$ detectable;

2. $D_{12}=\left[\begin{array}{c}0 \\ I_{m 2}\end{array}\right]$ and $D_{21}=\left[\begin{array}{ll}0 & I_{p 2}\end{array}\right]$ are then, respectively, of rank $m_{2}$ and $p_{2}$;

3. $\forall \omega \in \mathbb{R}$, rang $\left[\begin{array}{cc}A-j \omega I_{n} & B_{2} \\ C_{1} & D_{12}\end{array}\right]=n+m_{2}$ then this matrix is of full rank in addition $P_{12}$ has not invariant zeros on the imaginary axis;

4. $\forall \omega \in \mathbb{R}$, rang $\left[\begin{array}{cc}A-j \omega I_{n} & B_{1} \\ C_{2} & D_{21}\end{array}\right]=n+p_{2}$ then this matrix is of full rank in addition $P_{21}$ has not invariant zeros on the imaginary axis;

Let $D_{11}=\left[\begin{array}{ll}D_{1111} & D_{1112} \\ D_{1121} & D_{1122}\end{array}\right]$ with $D_{1122}$ is of dimension $m_{2} \times p_{2}$, the solution will be given by the following theorem :

\section{Theorem 1 :}

If a system $G(s)$ verifies the hypotheses 1 to 4 described above then :

a. it exists a controller $K(s)$ which stabilizes the closed-loop and such that $\left\|F_{l}(P, K)\right\|_{\infty}<\gamma$ if and only if :

1. $\gamma>\max \left(\bar{\sigma}\left[D_{1111} \quad D_{1112}\right], \bar{\sigma}\left[D_{1111} \quad D_{1121}\right]\right)$

2. it exists solutions $X \geq 0$ and $Y \geq 0$ which verify the two Riccati equations relating to Hamiltonians matrices $H$ and $J$, and such that: $\rho(X Y)<\gamma^{2}$, where $\rho($.$) design the$ spectral radius.

b. if the conditions of part a.) are satisfied, then the controllers which stabilise the system, and which verify $\left\|F_{l}(P, K)\right\|_{\infty}<\gamma$ are given by :

$$
K(s)=F_{l}(M, \phi)
$$

where $\|\phi(s)\|_{\infty}<\gamma$ and

$$
M=\left[\begin{array}{c|cc}
\hat{A} & \hat{B}_{1} & \hat{B}_{2} \\
\hline \hat{C}_{1} & \hat{D}_{11} & \hat{D}_{12} \\
\hat{C}_{2} & \hat{D}_{21} & 0
\end{array}\right]
$$

and

$\hat{D}_{11}=-D_{1121} D_{1111}^{T}\left(\gamma^{2} I_{m_{1}-P_{2}}-D_{1111} D_{1111}^{T}\right)^{-1} D_{1112}-D_{1122}$

$\hat{D}_{12} \in \mathbb{R}^{m_{2} \times m_{2}}$ and $\hat{D}_{21} \in \mathbb{R}^{p_{2} \times p_{2}}$ are two arbitrary matrices which verify :

$\hat{D}_{12} \hat{D}_{12}^{T}=I_{m_{2}}-D_{1121}\left(\gamma^{2} I_{m_{1}-P_{2}}-D_{1111}^{T} D_{1111}\right)^{-1} D_{1121}^{T}$

$\hat{D}_{21}^{T} \hat{D}_{21}=I_{p_{2}}-D_{1121}^{T}\left(\gamma^{2} I_{p_{1}-m_{2}}-D_{1111} D_{1111}^{T}\right)^{-1} D_{1121}$

$\hat{B}_{2}=Z\left(B_{2}+L_{12}\right) \hat{D}_{12}$ and $\hat{B}_{1}=-Z L_{2}+Z\left(B_{2}+L_{12}\right) \hat{D}_{11}$

$\hat{C}_{2}=-\hat{D}_{21}\left(C_{2}+F_{12}\right)$ and $\hat{C}_{1}=F_{2}-\hat{D}_{11}\left(C_{2}+F_{12}\right)$

$Z=\left(I_{n}-\gamma^{-2} Y X\right)^{-1}$

$\hat{A}=A+B F-\hat{B}_{1}\left(C_{2}+F_{12}\right)$ 
The controller calculated for $\phi(s)=0$ is called the Central Corrector, it is generally used under the form :

$$
K(s)=\left[\begin{array}{c|c}
\hat{A} & \hat{B}_{1} \\
\hline \hat{C}_{1} & \hat{D}_{11}
\end{array}\right]
$$

\section{References}

[1] G. N. Hingorani and L. Gyugyi, Understanding FACTS: concepts and technology of flexible AC transmission systems. Piscataway: IEEE Press, 2000.

[2] F. Goodrich and B. Andersen, The 2000 MW HVDC link between England and France. Power Engineering Journal Vol. 1, No. 2, pp 69-74, 1987.

[3] http://www.inelfe.eu/, INELFE Consortium

[4] http://www.rte-france.com/, Schéma décennal de développement du réseau, 2013.

[5] J. F. Hauer, Robustness issues in stability control of large electric power systems. Proc. 32nd IEEE Conference on Decision and Control, pp 2329-2334, 1993.

[6] N. A. Vovos and G. D Galanos, Enhancement of the Transient Stability of Integrated AC/DC Systems Using Active and Reactive Power Modulation IEEE Power Engineering Review, Vol. 5, No. 7, pp 33-34, 1985.

[7] T. Smed and G. Andersson, Utilizing HVDC to damp power oscillations IEEE Trans. Power Deliv, Vol. 8, No. 2, pp 620-627, 1993.

[8] A. E. Hammad, J. Gagnon and D. McCallum, Improving the dynamic performance of a complex AC/DC system by HVDC control modifications. IEEE Trans. Power Deliv, Vol. 5, No. 5, pp 1934-1943, 1990.

[9] F. L. Shun, R. Muhamad, K. Srivastava, S. Cole, D. V. Hertem and R. Belmans, Influence of VSC HVDC on transient stability: Case study of the Belgian grid. Proc. IEEE Power and Energy Society General Meeting, pp 1-7, 25-29 July 2010.

[10] C. W. Taylor and S. Lefebvre, HVDC controls for system dynamic performance. IEEE Trans. Power Syst, Vol. 6, No. 2, pp 743-752, 1991.

[11] H. F. Latorre, M. Ghandhari and L. Söder, Control of a VSC-HVDC operating in parallel with AC transmission lines. Proc. Transmission 83 Distribution Conference and Exposition IEEE, Latin America, pp 1-5, 2006.

[12] S. Henry, O. Despouys, R. Adapa and al, Influence of Embedded HVDC Transmission on System Security and AC Network Performance. Cigre, 2013.

[13] L. Arioua, B. Marinescu and E. Monmasson, Control of HVDC links with Overall Large-Scale Grid Objectives. IET Generation Transmission and distribution, Vol.8, No. 5, pp 945-956, 2014.

[14] Z. Hu, C. Mao and J. Lu, Improvement of transient stability in AC system by HVDC Light. Proc. Transmission and Distribution Conference and Exhibition IEEE, Dalian, pp 1-5, 2005. 
[15] C. Mao, Z. Hu, J. Lu, D. Chang and S. Fan, Application of an optimal coordinated control strategy to VSC HVDC. Proc. Power Systems Conference and Exposition IEEE, Atlanta, pp 2141-2145, 2006.

[16] B. Meyer, and M. Stubbe, EUROSTAG, a single tool for power system simulation. Transmission E Distribution International, 1992.

[17] L. Arioua and B. Marinescu, Implementation and Validation of Multivariable Control With Grid Objectives of an HVDC Link. Proc. Power Systems Computation Conference PSCC, Wroclaw, Poland, 2014.

[18] P. Kundur Power stability and control. New York: McGraw-Hill, 1994.

[19] G. Rogers, Power system oscillations. Boston: Kluwer Academic, 2000.

[20] T. L. Baldwin, L. Mili and A. G. Phadke, Dynamic Ward equivalents for transient stability analysis. IEEE Trans. Power Syst., Vol.9, No. 1, pp 59-67, 1994.

[21] H.F. Latorre, M. Ghandhari and L. Söder, Active and reactive power control of a VSC-HVDC. Electric Power Systems Research., Vol. 78, No. 10, pp 1756-1763, 2008.

[22] S. Skogestad and I. Postlethwaite Multivariable feedback control. Chichester: John Wiley, 1996.

[23] K. Zhou and J. C. Doyle Essentials of robust control. New Jersey: Prentice Hall, 1997.

[24] S. Li, T. A. Haskew and L. Xu, Control of HVDC Light System Using Conventional and Direct Current Vector Control Approaches. IEEE Trans. Power Electr, Vol. 25, No. 12, pp 3106-3118, 2010. 